\title{
Downregulation of CD10 in leukaemic phase of follicular lymphoma: a silent deception
}

\author{
Priya Mary Jacob • Rekha A Nair • Jayasudha A V • \\ Sindhu Nair P • Anila K R
}

Received: 11 September 2012 / Accepted: 2 January 2013 /Published online: 13 January 2013

(C) Springer-Verlag Berlin Heidelberg 2013

\begin{abstract}
Follicular lymphomas (FL) are frequent Bcell-derived malignancies, generally demonstrating an indolent evolution. Peripheral blood and bone marrow involvement by FL cells is infrequent. The aim of this study was to evaluate morphological features including immunophenotypic analysis of leukaemic follicular lymphoma on flow cytometry. The present study included 13 cases diagnosed on flow cytometry as follicular lymphoma involving peripheral blood or bone marrow over a period of 2 years (May 2010 to May 2012). Clinical presentation, complete blood count, peripheral smear, bone marrow aspirate and biopsy, lymph node biopsy and immunophenotypic profile by flow cytometric analysis of cases were reviewed. We observed in 13 patients a leukaemic phase of FL at the onset of the disease. Twelve of them had concomitant lymph node involvement whereas one patient had pure follicular cell leukaemia. In seven of 13 cases, there was absence of CD10 on flow cytometric analysis of circulating cells. Out of these seven cases, a lymph node biopsy was done in six cases, and all six cases showed CD10positive neoplastic germinal centres.
\end{abstract}

Keywords Follicular lymphoma $\cdot$ Peripheral blood $\cdot$ CD10

\section{Introduction}

Follicular lymphomas (FL) account for $20 \%$ of all nonHodgkin lymphomas (NHL). It affects predominantly adults with median age in the sixth decade. The bone

P. M. Jacob • R. A. Nair $(\bowtie) \cdot J$. A. V • S. N. P • A. K. R

Department of Pathology, Regional Cancer Centre,

Thiruvananthapuram, Kerala 695011, India

e-mail: drrekhanair@gmail.com marrow is involved in 40 to $50 \%$ cases [1]. FL cells typically express B cell antigens and CD10. However, absence of CD10 in the leukaemic phase has been observed, posing a practical challenge for correct diagnosis. Very few studies in literature have highlighted the fact that there is absence of CD10 in circulating FL cells and this can cause diagnostic confusion with other NHLs.

\section{Subjects and methods}

This is a retrospective study of 2 years duration from May 2010 to May 2012. A total of 13 cases in which a primary diagnosis of FL was made/suggested by flow cytometric immunophenotyping of peripheral blood or bone marrow were included. Bone marrow trephine biopsy was done in 12 out of 13 cases. A lymph node biopsy was done in nine cases for confirmation and was graded subsequent to this. Clinical details were obtained from the patient files and medical records.

Morphology

The peripheral blood and bone marrow aspirate smears were stained with Giemsa and myeloperoxidase stain for morphological evaluation. Of the 13 cases, flow cytometry (FCM) was done on peripheral blood in ten cases and in bone marrow in three cases. The classical morphological appearance of the cell in FL is a cleaved cell with through and through cleaving, anecdotally referred to as the buttock cell' (Fig. 1). In our study, majority of cases had a typical morphological appearance, except in four cases where there was a morphological differential diagnosis of a 'cleaved' chronic lymphocytic leukaemia (CLL) in two cases and marginal zone lymphoma in two cases. 


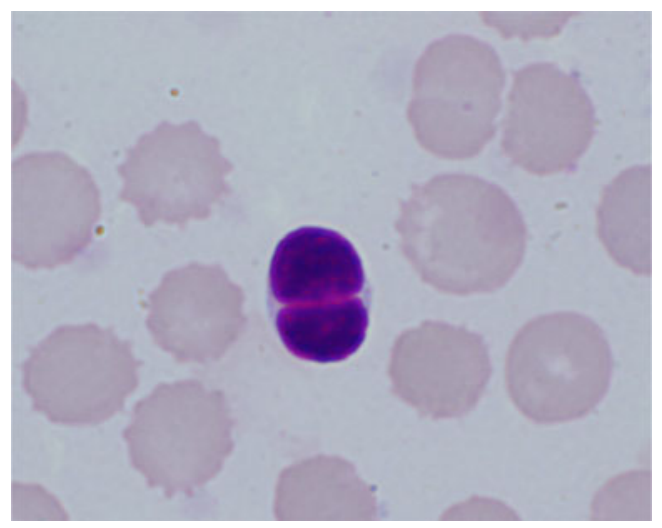

Fig. 1 Giemsa, $\times 1,000$ circulating tumour cells in FL

Immunophenotyping by flow cytometry

We analysed FCM data of these cases. Either the bone marrow aspirate or the peripheral blood sent in ethylenediaminetetraacetic acid was processed for immunophenotyping by FCM. The cells were prepared by whole blood stain, lyse and wash technique. Six-parameter, four-colour immunophenotyping was performed using a FACSCalibur (Becton Dickinson, San Jose, CA, USA). A minimum of 10,000 events were acquired using side scatter versus forward scatter gating. Data were analysed with CellQuestpro software (Becton Dickinson). Fluorochromes used were fluorescein isothiocyanate (FITC), phycoerythrin (PE), peridinin chlorophyll protein and allophycocyanin (APC). A panel of directly conjugated monoclonal antibodies, comprising of CD10 (FITC-HI10a), CD5 (PE-L17F12), CD23 (APCEBVCS-5), CD19 (PERCPr-4G7), CD20 (APC-L27), CD34 (PE-8G12), CD19 (APC-SJ25C1), CD45 (PERCP-2D1) and FMC7 (FITC-FMC7), were used. In selected cases, a few more antibodies were added which included kappa (PE-TB28-2), lambda (FITC-1-155-2) and CD38 (FITC-HB7).

\section{Results}

\section{Demography}

The age of patients in this study ranged from 25 to 74 years with median age at presentation being 53 years. The maleto-female ratio was $1: 1$.

Clinical features and immunoprofile

\section{Immunophenotypic features on flow cytometry}

Of the 13 cases studied, there were seven cases which were CD10 negative (Tables 1, 2 and 3). In these cases, the
Table 1 Clinical presentation

Feature at presentation

\begin{tabular}{ll}
\hline Leukocytosis $>11.0 \times 10^{9}$ & $5 / 13$ \\
Generalised lymphadenopathy & $9 / 13$ \\
Bulky abdominal lymph nodal mass & $2 / 13$ \\
Splenomegaly & $2 / 13$ \\
Age of youngest patient at diagnosis & 25 years \\
Median age & 53 years \\
\hline
\end{tabular}

neutrophil population was backgated to see if it was CD10 positive. This acts as an internal control as neutrophils normally express CD10 (Fig. 2). The cells determined to be cell surface CD10 negative were not subsequently interrogated using a permeabilization technique. In these cases, a diagnosis of FL was suggested as a differential diagnosis based on the morphology of the circulating tumour cells and the typical appearance of focal paratrabecular aggregates on bone marrow trephine biopsy. A lymph node biopsy was advised for confirmation and grading. Six cases were CD10 positive by flow cytometry. In these cases, a primary diagnosis of FL was made after taking into consideration, the morphology of circulating tumour cells and the immunophenotype. A lymph node biopsy was advised for grading (Table 4). While flow cytometry gives us the advantage of a quick diagnosis and a pointer towards the next step, a lymph node biopsy gives one the undeniable advantage of architecture where we can appreciate the follicular pattern and determine the grade.

\section{Bone marrow trephine biopsy}

A bone marrow trephine biopsy was done in 12 out of 13 cases. Specimens were received in Bouin's solution followed by decalcification in Gooding and Stewart's solution (10\% formic acid and $5 \%$ formaldehyde) and processed with other specimens to paraffin wax embedding. This is a modification of the Hammersmith protocol [2]. Among the 12 trephine biopsies, two were not representative. The remaining ten trephine biopsies

Table 2 Results of immunophenotyping by FCM along with intensity of antigen expression

\begin{tabular}{lll}
\hline Surface marker & No. of cases $(n=13)$ & Intensity \\
\hline CD20 & $13 / 13$ & +++ \\
CD10 & $6 / 13$ & + \\
CD23 & $7 / 13$ & + \\
FMC7 & $8 / 13$ & + \\
CD5 & $0 / 13$ & - \\
\hline
\end{tabular}


Table 3 Immunohistochemistry with CD10 on lymph node biopsy and bone marrow biopsy in cases of FL with loss of CD10 in circulation

\begin{tabular}{|c|c|c|c|c|}
\hline Case no. & $\begin{array}{l}\text { Lymph node } \\
\text { biopsy_diagnosis }\end{array}$ & $\begin{array}{l}\text { Lymph node biopsy-CD10 } \\
\text { expression in neoplastic } \\
\text { follicle }\end{array}$ & $\begin{array}{l}\text { Bone marrow } \\
\text { biopsy_diagnosis }\end{array}$ & $\begin{array}{l}\text { Bone marrow } \\
\text { biopsy-CD10 expression } \\
\text { in paratrabecular infiltrate }\end{array}$ \\
\hline 1. F, 44 years & FL grade I & Positive & Infiltration present & Negative \\
\hline 2. M, 53 years & FL grade I & Positive & Infiltration present & Negative \\
\hline 3. M, 62 years & FL grade II & Positive & Infiltration present & Negative \\
\hline 4. F, 41 years & FL grade III & Positive & Infiltration present & Negative \\
\hline 5. F, 63 years & FL grade III & Positive & Infiltration present & Negative \\
\hline 6. M, 51 years & $\begin{array}{l}\text { Not biopsied. Patient } \\
\text { has generalized } \\
\text { lymphadenopathy }\end{array}$ & Not done & $\begin{array}{l}\text { Biopsy done, not } \\
\text { representative }\end{array}$ & Not done \\
\hline 7. F, 44 years & FL grade II & Positive & Infiltration present & Focally positive \\
\hline
\end{tabular}

showed classical pattern of paratrabecular infiltration by small cleaved lymphoid cells.

\section{Lymph node biopsy with immunohistochemistry \\ (Tables 3 and 4)}

Among the seven cases which were CD10 negative by flow cytometry, a lymph node biopsy was done in six cases. It was not done in one case (case no. 6, Table 3) as the patient was not willing for the same (Table 3). CD10 (clone 56C6, Novocastra, Newcastle, UK: prediluted) by immunohistochemistry was positive in all six cases (Fig. 3a, b). Out of the six cases, two were grade I, two were grade 2 and two were grade 3 . Grade was determined on morphology by the method of counting large transformed cells (centroblasts) as described in WHO and supplemented by Molecular Immunology Borstal 1 labelling index. The interfollicular neoplastic cells were variably CD10 positive or negative.

Nine out of 13 cases required therapy, with a short interval between diagnosis and treatment initiation. Rituximab-based regimens were used, in combination with cyclophosphamide, doxorubicin, vinchristine, prednisone (CHOP) in four cases. In another four cases, CHOP was given without rituximab, as they could not afford the same. One patient could not tolerate chemotherapy and was started on oral drugs endoxan and prednisone. The median followup since diagnosis was 20 months (3 to 34 months). All patients who were treated experienced complete response and are stable on follow-up. Four patients who were not treated are on regular follow-up and are asymptomatic.
Fig. 2 Flow cytometric dot plots in a case of FL with loss of CD10. The neutrophils were backgated (in green) as an internal control for CD10
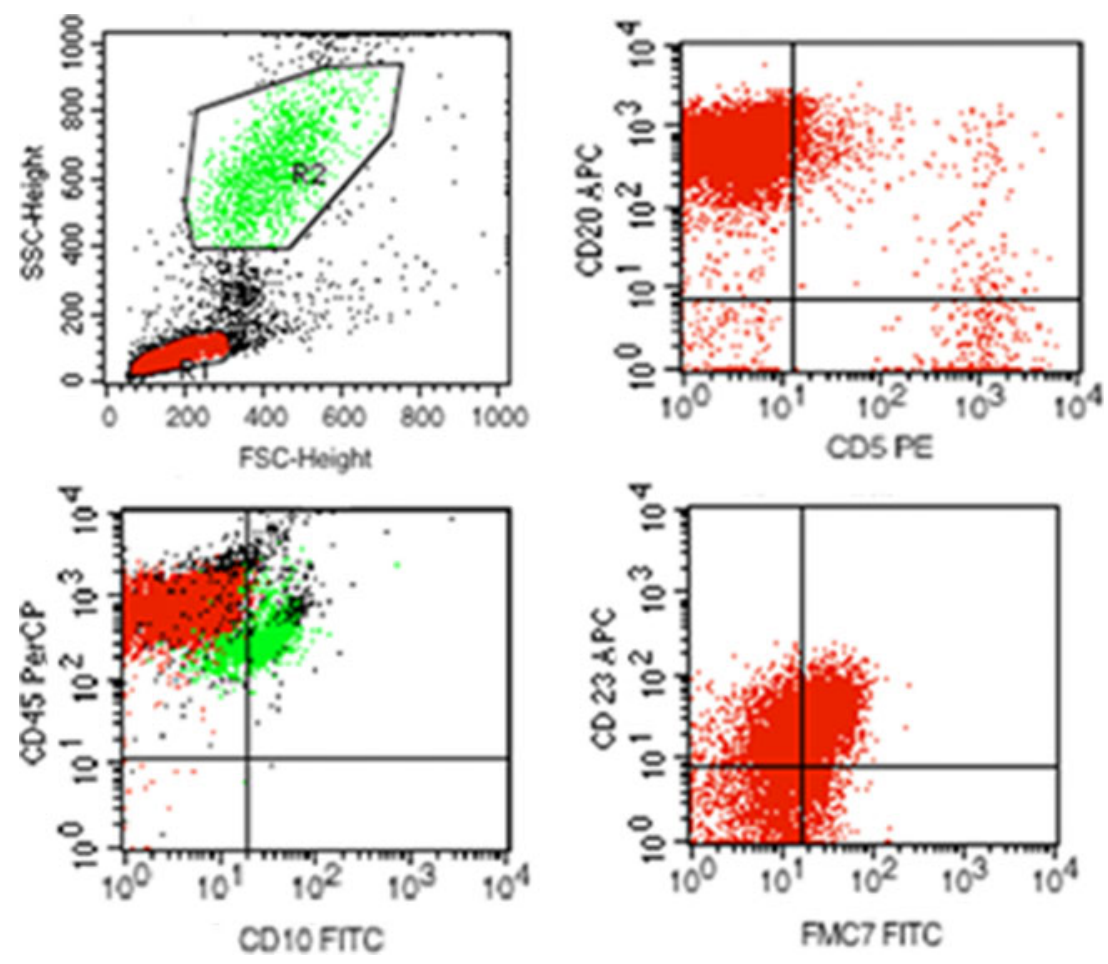
Table 4 Lymph node and bone marrow biopsy diagnosis in remaining six cases of FL which were CD10 positive by flow cytometry in circulation

\begin{tabular}{|c|c|c|}
\hline Case no. & Lymph node biopsy_diagnosis & Bone marrow biopsy-diagnosis \\
\hline 8. F, 38 years & FL grade I & Infiltration present \\
\hline 9. M, 74 years & $\begin{array}{l}\text { Lymph node biopsy not done. Fine needle aspiration } \\
\text { cytology_-low-grade FL }\end{array}$ & Infiltration present \\
\hline 10. F, 63 years & $\begin{array}{l}\text { Composite lymphoma-FL grade I and nodal } \\
\text { marginal zone lymphoma }\end{array}$ & Infiltration present \\
\hline 11. F, 25 years & FL grade III & Not representative \\
\hline 12. M, 50 years & $\begin{array}{l}\text { No lymphadenopathy. CT scan of neck, chest, abdomen } \\
\text { and pelvis was done. De novo follicular cell leukaemia }\end{array}$ & Infiltration present \\
\hline 13. M, 69 years & $\begin{array}{l}\text { Not biopsied. Patient has a } 7 \times 3-\mathrm{cm} \text { lymph nodal mass } \\
\text { encasing branches of superior mesenteric artery }\end{array}$ & Not done \\
\hline
\end{tabular}

\section{Discussion}

The leukaemic phase of FL has been described in literature, but very few studies have highlighted the absence of membrane expression of CD10 in the leukaemic phase. In a study by Al-Nawakil et al., out of ten cases, one case showed loss of CD10 and one showed low expression of CD10. These were considered as possible FL [3]. In our study, in seven out of 13 cases, there was absence of CD10 on flow cytometric analysis of circulating cells. Out of these seven cases, a lymph node biopsy was done in six cases, and all six cases showed CD10positive tumour cells. Given the different methodologies (flow cytometry versus immunohistochemistry) used for CD10 staining, the possibility of technical factors leading to this disparity was considered. In the paraffin section, immunohistochemical staining of CD10 is localized to the membrane of tumour cells. The flow cytometric technique is designed to optimize antibody reactivity with surface antigens [4]. In this study, as the cells determined to be cell surface CD10 negative by flow cytometry were not interrogated using a permeabilization technique, this could be a reason for disparity between the two staining techniques. To counter this argument, immunohistochemistry for CD10 was done on bone marrow trephine biopsies of those cases which were CD10 negative by flow cytometry (Table 3 ). Bone marrow biopsies of six out of seven cases were stained with immunohistochemical stains for CD10, keeping trephine biopsy of a CALLA-positive B acute lymphoblastic leukaemia which was fixed, decalcified and processed similarly, as a positive control. In one case, the bone marrow trephine was too scanty and so was not included. Five out of six bone marrow trephine cases were CD10 negative by immunohistochemistry, while one case was focally CD10 positive. These data illustrate that there is downregulation of $\mathrm{CD} 10$, once the tumour cells from the lymph node reach the circulation (Fig. $4 \mathrm{a}, \mathrm{b}$ ).

FL which is CD10 negative on flow cytometry is an entity which can easily be confused with other low-grade B cell lymphomas in a leukaemic phase. In CLL, 'cells with nuclear clefts' have been described by Criela et al. and Molica and Alberti [5, 6] and Criela et al. and Vallespi et al. [5, 7]. Some cases of CLL have an atypical immunophenotype (CD5- or CD23-, FMC7+ or CD11c+, strong sIg or CD79b+) [5]. This when accompanied by cleaving of tumour cell can lead to confusion with cases of FL with absence of CD10. Tumour cells of marginal zone lymphoma are CD20+, CD79a+, CD5-, CD10-, CD23- and CD43-. Absence of CD10 helps to exclude FL [1]. In our study, in two cases, the morphology of FL cells resembled marginal zone lymphoma cells with abundant cytoplasm and round nuclei with condensed chromatin. This unusual morphology if coupled with absence of CD10 on flow cytometry has potential for diagnostic confusion. Zach Liu et al. describe a cohort of mantle cell lymphoma cases without detectable CD5 expression, which may be a potential pitfall for this diagnosis. Lymphoma cells in PB samples in cases of centrocytic mantle cell lymphoma are small to medium lymphocytes with moderately clumped chromatin and
Fig. 3 a Haematoxylin and eosin staining $\times 400$. Neoplastic germinal centre of FL grade 2 showing centroblasts and centrocytes; b CD10, $\times 100$. CD10-positive neoplastic germinal centre cells of the same case
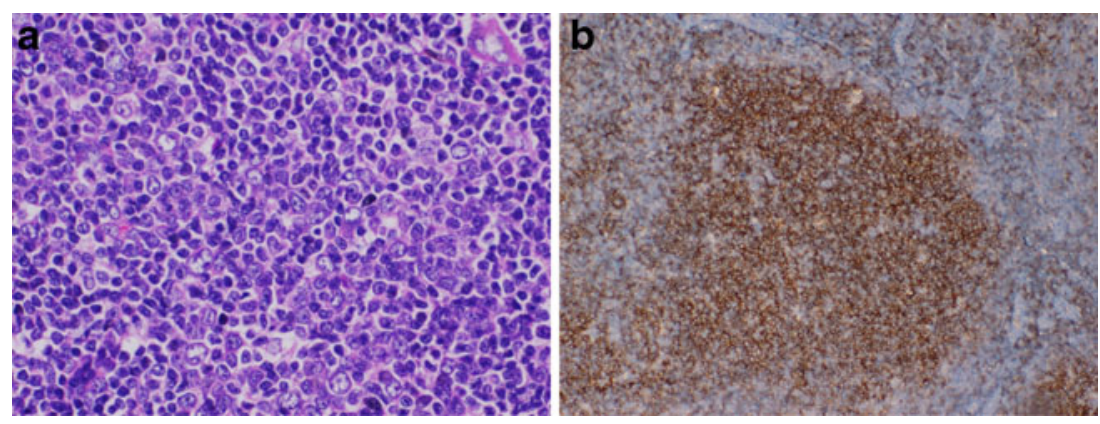
Fig. 4 a $H \& E, \times 400$. Bone marrow biopsy with characteristic paratrabecular infiltration. b CD10, $\times 400$. The bone marrow infiltrate is CD10 negative. Note that background neutrophils are CD10 positive
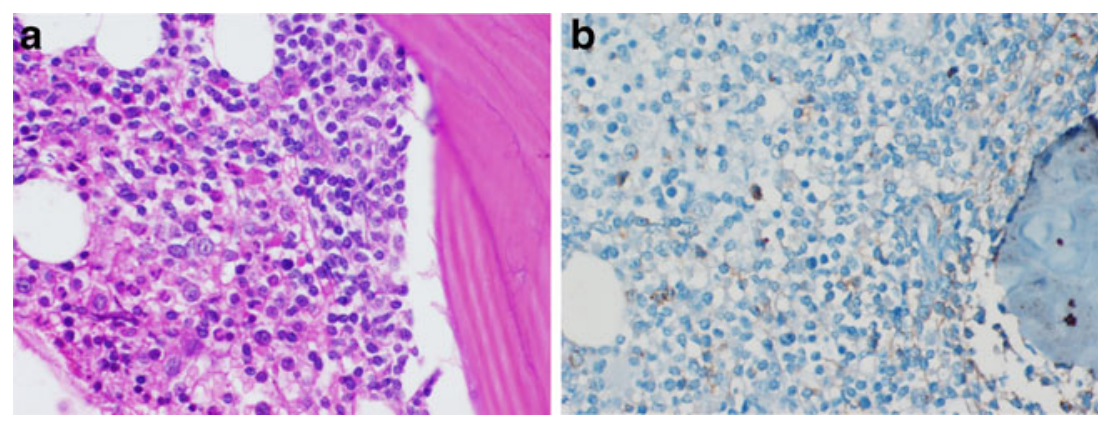

irregular, indented and cleaved nuclear contours and can thus closely resemble those of FL cells. Undue reliance on one criterion (such as CD5 expression by B cells) may lead to an erroneous diagnosis. Evaluation of cyclin D1 expression by immunohistochemical analysis or molecular genetics is needed if mantle cell lymphoma is suspected [8]. The CD23/FMC7 patterns in these entities may be useful to differentiate them. In FL, although variable patterns are described (CD23 \pm /FMC7+, $\mathrm{CD} 23 / \mathrm{FMC} 7+, \mathrm{CD} 23 \pm / \mathrm{FMC} 7 \pm, \mathrm{CD} 23+/ \mathrm{FMC} 7+), \mathrm{FL}$ is more likely to have the $\mathrm{CD} 23+/ \mathrm{FMC} 7+$ pattern [9].

A review of literature was done to understand the role of CD10 in haematopoietic cells and to seek answers for the loss or silencing of CD10 once the same tumour cell enters the circulation. Neutral endopeptidase or CD10 is a 90- to 110$\mathrm{kDa}$ zinc-dependent cell-surface metallopeptidase that cleaves peptide bonds on the amino side of hydrophobic amino acids. Initially identified as the common acute lymphoblastic leukaemia antigen, CD10 is normally expressed by early-B, pro-B and pre-B lymphocytes and by lymph node germinal centres, reflecting a biphasic pattern of CD10 expression in B cell differentiation. Expression of CD10 by leukaemic cells is indicative of the differentiation stage of normal lymphopoiesis from which the malignant cell derives [10, 11]. Thus, CD10 is found on non-T cell ALL cells which derive from pre-B lymphocytes and on germinal centre-related NHL such as Burkitt lymphoma and FL, but not on leukaemic cells or lymphomas, which originate in more mature B cells. In addition to normal germinal centres, CD10 is also expressed in neutrophils [12] and early B cell precursors (haematogones) within the bone marrow [13]. Some cases of FL, especially grade 3B, may lack CD10 but retain BCL6 expression [1]. CD10 expression is often stronger in the follicles than in interfollicular neoplastic cells and maybe absent in the interfollicular compartment as well as in areas of marginal zone differentiation, in peripheral blood and in bone marrow. In a study by Dogan et al., the immunophenotype of the follicular and interfollicular tumour cells was compared. They observed that the interfollicular B neoplastic cell component had lowgrade cytological features and low proliferation fraction and showed downregulation/absence of activation markers like CD10, CD38, CD95 and T cell co-stimulatory molecules CD80 and CD86. The presence of such a resting (CD10 negative) tumour subpopulation could provide a reservoir of tumour cells for the progression of FL after treatment. Conceivably, it is these cells that constitute the optimum target for the treatment of FL [14]. The presence of such a neoplastic subpopulation within the lymph node could explain the origin of these CD10-negative circulating tumour cells. CD10 is developmentally regulated in both hematopoietic and epithelial progenitor cells. Two separate regulatory regions type 1 and type 2 neutral endopeptidase (NEP) control the transcription factor binding sites. Dense methylation of the type 2 NEP regulatory region had suggested that methylation may play a role in controlling CD10 transcription [10, 15]. Papandreou and Nanus and Ikawa et al. now provide convincing evidence that methylation of transcription factor binding sites contributes to CD10 silencing $[10,16]$. CD10 possesses multiple biologic effects. CD10's cytoplasmic domain directly interacts with a variety of proteins, including the phosphatase and tensin homolog (PTEN) protein $[10,17,18]$. CD10 recruits endogenous PTEN to the cell membrane, leading to prolonged PTEN protein stability and increased PTEN phosphatase activity, resulting in constitutive downregulation of protein kinase $\mathrm{B}$ activity. Consequently, in epithelial cells, CD10 loss from methylation leads to increased cell migration, cell growth and cell survival, contributing to neoplastic development and progression $[10,19]$. This mechanism of epigenetic silencing by methylation may explain why some cases of FL show absence of $\mathrm{CD} 10$ once the tumour cells reach the circulation. We can also raise the possibility of a particular pattern of expression of membrane antigens such as adhesion molecules on the surface of circulating FL cells, as suggested to explain the circulating component of plasma cells in primary plasma cell leukaemia compared to classical plasma cell myeloma [3]. The lack of CD56 antigen, a neural adhesion molecule which is important in anchoring plasma cells to the bone marrow stroma, preventing circulation of myeloma cells to peripheral blood, as well as their migration to extramedullary sites, is a frequent finding in plasma cell leukaemia [20]. The precise role of CD10 on hematopoietic cells is not completely known but presumably is similar to other cell types. Whether CD10 (or lack thereof) is more than just a marker of prognosis or a target for therapy such as using demethylating agents to restore CD10 expression will require further study [10].

In our study, we observed in 13 patients a leukaemic phase of FL at the onset of the disease. Twelve of them had 
concomitant lymph node involvement whereas in one patient (case12), there was no lymph node enlargement on clinical examination. CT scan of neck, chest, abdomen and pelvis of this patient revealed no lymphadenopathy. A diagnosis of follicular cell leukaemia was made based on characteristic morphological appearance of cells (small cleaved atypical lymphoid cells with condensed chromatin) on BMA, typical immunoprofile by flow cytometry and the bone marrow biopsy which showed a paratrabecular aggregate of atypical small lymphoid cells. The patient is presently asymptomatic and on follow-up. A study by C. Al-Nawakil et al. represents the largest series of de novo leukaemic phase of FL. Four out of ten patients presented as pure follicular cell leukaemia, which has not yet been reported in literature so far, although a similar clinical entity is described in mantle cell lymphoma. In their series, the occurrence of pure follicular cell leukaemia appeared to be associated with a favourable outcome [19].

\section{Conclusion}

This study attempts to highlight the downregulation of CD10 expression in the leukaemic phase of seven out of 13 cases of FL. This circulating CD10-negative tumour population could possibly trace its origin to the interfollicular component of a FL. The topic is an important one, given the prevalence of FL in our population and the potential for diagnostic confusion with other low-grade B cell lymphomas with a leukaemic component. In the future, should some targeted therapeutic modality be developed against $\mathrm{CD10}$, the absence of this marker in the leukaemic phase could be a possible contraindication to the use of the prospective drug. With flow cytometric and immunohistochemical studies becoming the standard of care in diagnosing lymphoma, it is important to recognize this variation of CD10 expression and the limitations of flow cytometric and immunohistochemical analysis, especially when only one modality is used for immunophenotyping.

Conflict of interest The authors declare that they have no conflict of interest.

\section{References}

1. Harris NL, Swerdlow SH, Jaffe ES, Ott G, Nathwani BN et al (2008) Follicular lymphoma. In: WHO classification of haematopoietic and lymphoid tissue. Lyon, France, pp 220-226

2. Naresh KN, Lampert I, Hasserjian R, Lykidis D, Elderfield K, Horncastle D, Smith N, Murray-Brown W, Stamp GW (2006) Optimal processing of bone marrow trephine biopsy: the
Hammersmith Protocol. J Clin Pathol 59:903-911. doi:10.1136/ jcp.2004.020610

3. Al-Nawakil C, Kosmider O, Stern M-H, Manie E et al (2011) Leukemic phase of follicular lymphomas: an atypical presentation. Leukemia \& Lymphoma 52(8):1504-1508

4. Kurtin PJ, Hobday KS, Ziesmer S, Caron BL (1999) Demonstration of distinct antigenic profiles of small B-cell lymphomas by paraffin section immunohistochemistry. Am J Clin Pathol 112(3):319-29

5. Criela A, Michauxb L, de Wolf-Peeters C (1999) The concept of typical and atypical chronic lymphocytic leukaemia. Leukemia and Lymphoma 33(1-2):33-45

6. Molica S, Alberti A (1988) Investigation of nuclear clefts as a prognostic parameter in chronic lymphocytic leukemia. Eur J Haematol 41:62-65

7. Vallespi T, Montserrat E, Sam MA (1991) Chronic lymphocytic leukaemia: prognostic value of lymphocyte morphological subtypes. A multivariate survival analysis in 146 patients. British Journal of Haematology 77:478-485

8. Liu Z, Dong HY, Gorczyca W, Tsang P, Cohen P, Stephenson CF, Berger CS, Wu CD, Weisberger J (2002) CD5- mantle cell lymphoma. Am J Clin Pathol 118:216-224

9. Garcia DP, Rooney MT, Ahmad E, Davis BH (2001) Diagnostic usefulness of CD23 and FMC-7 antigen expression. Patterns in B-cell lymphoma classification. Am J Clin Pathol 115(2):258-265

10. Papandreou CN, Nanus DM (2010) Is methylation the key to CD10 loss? Journal of Pediatric Hematology/Oncology 32(1):23. doi:10.1097/MPH.0b013e3181c74aca

11. Bene MC (2005) Immunophenotyping of acute leukaemias. ImmunolLett 98:9-21

12. MA Shipp, GB Stefano, SN Switzer, JD Griffin, EL Reinherz (1991) CD10 (CALLA)/neutral endopeptidase 24.11 modulates inflammatory peptide- induced changes in neutrophil morphology, migration, and adhesion proteins and is itself regulated by neutrophil activation. Blood 78(7):1834-1841

13. McKenna RW, Washington LT, Aquino DB, Pickerand LJ, Kroft SH (2001) Immunophenotypic analysis of hematogones (B-lymphocyte precursors) in 662 consecutive bone marrow specimens by 4-color flow cytometry. Blood 98(8):2498-2507

14. Dogan A, Du MQ, Aiello A, Diss TC, Ye HT, Pan LX, Isaacson PG (1998) Follicular lymphomas contain a clonally linked but phenotypically distinct neoplastic B-cell population in the interfollicular zone. Blood 91(12):4708-4714

15. Taylor KH, Liu J, Guo J et al (2006) Promoter DNA methylation of CD10 in lymphoid malignancies. Leukemia 20:1910-1912

16. Ikawa K, Sugimoto N, Koizumi S et al (2010) Dense methylation of type 1 and type 2 regulatory regions of theCD10 promoter in infant acute lymphoblastic leukemia with MLL/AF4 fusion gene. J PediatrHematolOncol 32:4-10

17. Sumitomo M, Iwase A, Zheng R et al (2004) Synergy in tumor suppression by direct interaction of neutral endopeptidase with PTEN. Cancer Cell 5:67-78

18. Sumitomo M, Shen R, Walburg M et al (2000) Neutral endopeptidase inhibits prostate cancer cell migration by blocking focal adhesion kinase signaling. J Clin Invest 106:13991407

19. Sumitomo M, Shen R, Nanus DM (2005) Involvement of neutral endopeptidase in neoplastic progression. Biochim Biophys Acta 1751:52-59

20. Al-Nawakil C, Tamburini J, Bardet V et al (2008) Bortezomib, doxorubicin and dexamethasone association is an effective option for plasma cell leukemia induction therapy. Leuk Lymphoma 49:2012-2014 